BULL. AUSTRAL. MATH. SOC.

VOL. $29(1984), 157-158$.

\title{
TIME SERIES IN MEDICAL RESEARCH
}

\author{
LESLIE J. HILLS
}

The National Trachoma and Eye Health Program (1980) has a section reporting the prevalence of midale ear infection (otitis media) in aboriginal communities. As perforation of the eardrum normally follows the infection at the level being considered, scars remain as in indication of past infection.

A semi-Markov model is a suitable model for describing medical case history, where each subject is classified as being in one of several states and there are hazards of transition from state to state. Here there are two states, infected and not infected, and the hazards are thus: the hazard of becoming infected or incidence rate of the disease, and the hazard of recovery or recovery rate.

Initially we consider a model of constant recovery rate, with incidence a function of age. However, it is clear that there is nonstationarity and a covariable is introduced, this being the time lapse since a subject was a given age. In effect this allows for changing incidence of the disease over time.

It is then considered that a subject may have lost scars from previous infection and this was built into the model, first as an alternative to, then together with, changing incidence. The fading scars proposition seemed to help in fitting the data for non-aborigines as the lower incidence allowed time for this to occur, but changing incidence

Received 16 April 1984. Thesis submitted to the University of New South Wales, February 1983. Degree approved February 1984.

Supervisor: Associate Professor C. A. McGilchrist.

Copyright clearance centre, Inc. Serial-fee code: 0004-9727/84 $\$ A 2.00+0.00$ 
seems to largely account for the non-stationarity of the aboriginal data. It appears that the aboriginal people are becoming increasingly subject to an infection of comparatively minor importance among non-aborigines.

In Section 2, data from the Diet-Heart Study by Professor Blacket (Department of Medicine, Prince Henry Hospital, Sydney) is under consideration. The purpose is to describe changes in serum cholesterol in terms of dietary factors, and the subjects were patients recovering from coronary disease. These patients were assessed six-monthly over varying periods of time, so that we had a large number of short time series.

The model appropriate to this data is a regression model with a correlated error structure over time. In order to use all series for parameter estimation and yet still have individual estimates for each subject, Bayesian methods are used. Also to facilitate computations and aid in model diagnostics, techniques of recursive estimation are extended to the model being considered.

We first find likelihood function and recursive residuals for the stationary error model, then investigate the regression model, and finally introduce the Bayesian methods. When the data is fitted we find that serum cholesterol changes are to a large extent explained by changes in weight of the subject, and diet cholesterol changes do not appreciably add to the model's ability to explain the data.

School of Mathematical Sciences,

The New South Wales Institute of Technology, P.O. Box 123,

Broadway,

New South Wales 2007, Australia. 